Article original

\title{
Tumeur fibreuse pleurale gauche géante en situation clinique
}

\author{
Malika Metahri, Abdelmadjid Snouber, Nedjma Mened
}

Service de pneumophtysiologie A-CHU Oran

\section{Introduction}

La tumeur fibreuse pleurale solitaire, longtemps considérée fibrome pleural ou mésothélium fibreux localisé, est une tumeur mésenchymateuse rare, représentant 5\% des masses pleurales. Le premier cas a été rapporté en 1931 par Klemperer et Rabin; 800 cas sont rapportés dans la littérature jusqu'à 2010, dont deux grandes séries: Briselli (USA, 1981, 360 cas et England (USA, 1989) ,223 cas, les autres séries ne dépassant pas 30 cas[1]. Elle est réputée bénigne, souvent bien limitée, encapsulée, guérissant après exérèse complète.

\section{Observation}

Patiente A.B. âgée de 38 ans, originaire et demeurant à Tiaret, sans antécédents pathologiques, admise pour investigation d'une dyspnée et douleurs thoraciques gauches avec images radiologique et scannographique pathologiques.

Le début remontait à 04 mois par une toux sèche, dyspnée stade Il et douleur thoracique, d'où son hospitalisation à Tiaret pendant un mois pour suspicion d'une pleurésie gauche de grande abondance. Plusieurs ponctions ont été tentées sans succès, ce qui a motivé son orientation vers notre service.

L'état général était conservé, l'examen pleuro-pulmonaire notait une matité diffuse de l'hémithorax gauche, avec abolition du murmure vésiculaire et des vibrations vocales. Le reste de l'examen clinique était sans anomalies. La radiographie thoracique de face (fig.1) montrait une opacité dense, homogène, prenant la totalité de l'hémithorax gauche, refoulant le médiastin vers la droite, n'effaçant pas totalement l'hémicoupole gauche. Le bilan biologique standard était normal.

L'intradermo-réaction à la tuberculine, la recherche du bacille de Koch dans les crachats, et la sérologie hydatique étaient négatives. La fibroscopie bronchique montrait une compression et un refoulement de tout l'arbre bronchique gauche. Le diagnostic de pleurésie enkystée a été retenu sans écarter une éventuelle étiologie tumorale sous jacente.

La tomodensitométrie thoracique a objectivé un volumineux processus tissulaire, d'origine pleurale mesurant $15,3 \mathrm{~cm} \times 13,4 \mathrm{~cm}$, se raccordant en pente douce avec la paroi, hétérogène, par la présence d'hypodensités, se rehaussant après injection de produit de contraste. Cette masse refoulait le médiastin vers le côté controlatéral et comprimait le parenchyme pulmonaire qui était totalement collabé.Le diagnostic d'un processus tumoral pleural est retenu (fig. 2 et 3).

Une biopsie transpariétale a été réalisée, l'examen anatomopathologique montrait une prolifération tumorale de densité cellulaire élevée, à cellules fusiformes, à noyau allongé parfois vacuolaire mais dépourvu de mitoses. Ces cellules se disposent en faisceaux enchevêtrés avec de nombreuses zones de sclérose hyaline. Cet aspect histologique est celui d'un mésothéliome pleural dans sa forme fibreuse. 


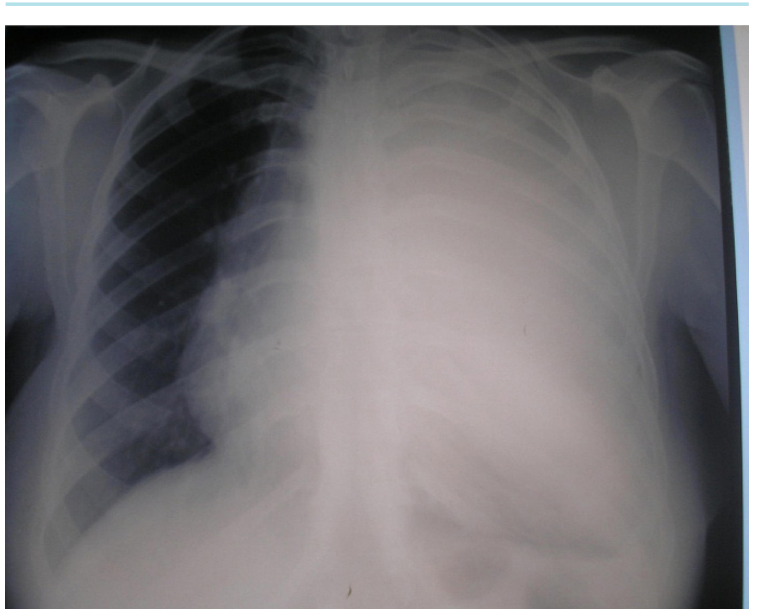

Figure 1. Radiographie thoracique de face : À gauche Hémithorax sombre refoulant n'effaçant pas totalement l'hémi coupole gauche

Une thoracotomie postéro-latérale par le 5ème espace intercostal gauche, montrait, dès l'ouverture pleurale, une masse occupant la totalité de l'hémithorax, refoulant le poumon en dedans. Elle semblait à point de départ de la plèvre viscérale. L'exérèse complète de la tumeur a été réalisée, mais la patiente décédait des suites opératoires immédiates.
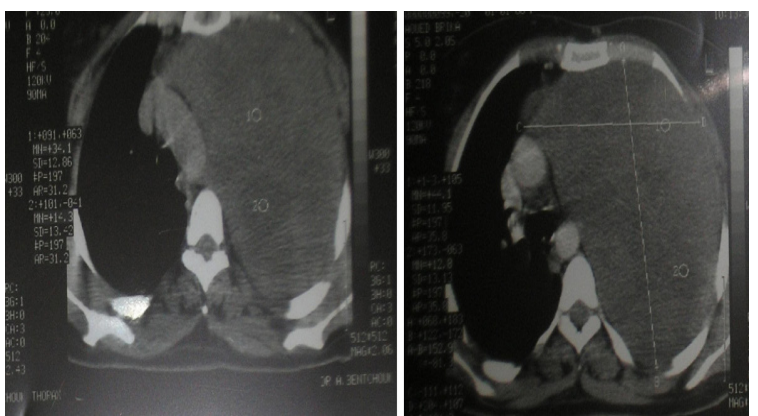

Figure 2 (a, b). TDM thoracique: (fenêtre médiastinale) Volumineuse masse tissulaire avec zones de nécrose en contact étroit avec la crosse de l'aorte, refoulant le médiastin vers le côté opposé.
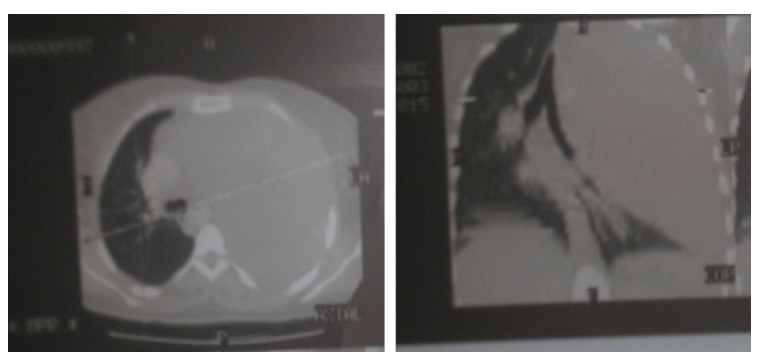

Figure 2 (a, b).TDM thoracique: (fenêtre médiastinale) Volumineuse masse tissulaire avec zones de nécrose en contact étroit avec la crosse de l'aorte, refoulant le médiastin vers le côté opposé.

\section{Discussion}

La tumeur fibreuse pleurale, est souvent une tumeur unique bien localisée, qui représente $5 \%$ des tumeurs de la plèvre [2]. L'âge de survenue de ces tumeurs est compris entre 30 et 70 ans avec une moyenne de 53,3 ans [3]. On ne note aucune prédominance de sexe, aucun facteur favorisant n'a pu être individualisé en particulier une exposition professionnelle.

Plusieurs appellations sont données à cette tumeur : Le fibrome pleural localisé bénin, le mésothéliome fibreux localisé de la plèvre, le fibrome pleural sous mésothélial, le mésothéliome fibreux localisé, et tumeur fibreuse localisée bénigne de la plèvre.

Sur le plan clinique, cette tumeur est souvent asymptomatique de découverte radiologique fortuite. Rarement elle se manifeste comme chez notre patiente, par des signes respiratoires de type dyspnée, toux ou douleurs thoraciques ; parfois une symptomatologie neurologique du membre supérieur, par compression du plexus brachial peut être notée dans les tumeurs de siège apical [4].

Un épanchement liquidien peut être retrouvé mais généralement minime, des manifestations extra-thoraciques peuvent être révélatrices de ces tumeurs, en particulier deux tableaux sont fréquents :

- L'ostéo-arthropathie hypertrophiante de Pierre Marie retrouvée dans $16 \%$ des cas [3].

- Des accès d'hypoglycémie retrouvés dans $4 \%$ des cas dont les anomalies des IGF-I et -II (insulin-likegrowth factor) et de leurs protéines porteuses semblent être l'élément clé de la pathogénie [5].

Par ailleurs, un hippocratisme digital et une acné faciale ont été rapportés [3], ayant disparu deux mois après l'exérèse de la tumeur.

Ces syndromes paranéoplasiques sont surtout l'apanage des tumeurs volumineuses dont le diamètre dépasse $7 \mathrm{~cm}$ [3].Ces aspects n'ont pas été retrouvés chez notre patiente malgré un volume tumoral important.

A la radiographie thoracique on note souvent une opacité homogène, bien limitée dont la taille est variable, le siège de la tumeur est aussi très variable, et peut être apical, médiastinal, axillaire, diaphragmatique, inter-scissural, au niveau de la gouttière hosto-vertébrale, voire même tout l'hémichamps, comme chez notre patiente.

La tomodensitométrie thoracique apporte des renseignements importants pour le diagnostic en montrant une formation tissulaire en rapport avec les éléments du médiastin et avec la paroi qui est souvent indemne de toute atteinte ; elle permet de préciser son origine pleurale et d'éliminer un éventuel kyste hydatique, un épanchement cloisonné ou un lipome et ceci par 
rehaussement après injection de produit de contraste.

Le diagnostic est fait par biopsie transpariétale ou l'examen anatomopathologique de la pièce d'exérèse [6].

Sur le plan macroscopique, la tumeur est souvent volumineuse mais non infiltrante, lisse, parfois bosselée, bien encapsulée [7, 8]. Souvent unique, parfois il s'agit d'une double localisation ou l'association d'une tumeur et de multiples nodules à distance. Il n'existe aucune prédominance pour un lobe donné. En ce qui concerne l'attache de la tumeur, elle se fait cinq fois plus avec la plèvre viscérale $[3,7]$.

Al'histologie, la tumeur est faite d'une prolifération de cellules fusiformes de nature mésenchymateuse avec des fibres de collagène en abondance. L'analyse précise de la tumeur permet de retrouver certains constituants de façon constante avec des proportions variables $[3,9,10]$ :

- Des cellules fusiformes disposées en îlots,

- Des fibres collagènes prenant 3 aspects possibles :

- De fins filaments, des trousseaux allongés ou de volumineux blocs hyalinisés.

- Une richesse vasculaire.

- Une surface tumorale caractérisée par une condensation de fibres de collagène en périphérie et un liséré de cellules mésothéliales dans $2 / 3$ des cas.

- Des remaniements (myxoïde, nécrose, hémorragies, infiltrats inflammatoires) sont occasionnels.

Ainsi, certains auteurs [3, 9] insistent sur l'absence de mitoses, sur l'infiltration de la capsule juxta-pulmonaire, sur l'absence de valeur pronostique d'une discrète anisocaryose, sur les remaniements myxomateux [10].

Le traitement du mésothéliome fibreux de la plèvre est exclusivement chirurgical $[3,7,12]$. L'exérèse de la tumeur est souvent sans problème après sa libération du parenchyme pulmonaire qui est uniquement refoulé et collabé. En cas d'atteinte parenchymateuse par des phénomènes inflammatoires de voisinage, des résections parenchymateuses très limitées peuvent être réalisées. Certains cas de pneumonectomies ont été rapportés dans la littérature [13], vu que la tumeur avait un volume important, occupant le parenchyme, le rendant non réexpansible. Les récidives locales sont possibles et les métastases fibro-sarcomateuses sont exceptionnellement rapportées $[3,11,14]$. Ces dernières sont l'apanage des tumeurs volumineuses incomplètement réséquées. Une embolisation pré-opératoire est recommandée dans les tumeurs fibreuses géantes. Le pronostic de ces tumeurs opérées reste très bon avec une évolution très favorable sans récidives dans $88 \%$ des cas [3]. Non opérées, leur évolution est très lente, pouvant atteindre des volumes importants, responsables de trouble de la ventilation par compressions et de difficultés opératoires. Par ailleurs, il est possible de rencontrer ces tumeurs dans des sites extrapleuraux, tels que, les méninges, l'orbite, la sphère ORL et le tractus uro-génital. La symptomatologie est parfois aspécifique, marquée par des douleurs thoraciques, dyspnée, toux, trouble du rythme cardiaque et compression de la veine cave.

Notre observation est caractérisée par la présence de signes cliniques (dyspnée, toux et douleur thoracique), par le caractère géant de la tumeur (occupant plus de $40 \%$ de l'hémithorax) dont la largeur était supérieure à $10 \mathrm{~cm}$, la présence de zones de nécrose, le rehaussement après injection de produit de contraste, le contact étroit avec l'aorte et le refoulement du médiastin. Ces caractéristiques constitueraient des arguments de mauvais pronostic faisant partie des critères d'England [9] ; sachant que 20 à $40 \%$ des tumeurs fibreuses pleurales solitaires sont cliniquement ou histologiquement malignes.

\section{Conflit d'intérêt}

Les auteurs déclarent n’avoir aucun intérêt.

\section{Références bibliographiques}

[1] J A Pinedo-Onofre, E Robles-Pérez, ES Pena-Mirabal,J A Hernandez-Carillo,J L Téllez-Becerra.Giant Solitary fibrous tumor of the pleura.Cir Ciruj 2010;78:31-42

[2] M'RAIHI. M.L, SANSONETTI.M, GARBE L., FUENTES P. ,SEISdEDOS M.C., VERVLOET D.Le fibrome pleural : A propos d'un cas.Rev. Mal. Resp., 1990, 7, 79-82

[3] LOIRE R, PINET-ISAAC S., AL HAMANY. Z, REVEL D., TABIBA., CORDIER J.F.Le mésothéliome fibreux pleural localisé (fibrome pleural sous-mésothélial) étude anatomo-clinique de 25 cas. Ann, Pathol. 1992, 12, $n^{\circ} 2,102-108$

[4] BAZELLY B., TERRIOUX PH., FRANÇOIS TH., BLONCHON F.Une pathologie souvent trompeuse : le fibrome pleural, à propos de deuxcas récents de diagnostic opératoire.Sem. Hôp. Paris, 1984, 60, 1267-70

[5]M. Hamidou, F. Bani-Sadr, A. Kenzi, C. Sagan, J.Y. Grolleau Hypoglycémie associée aux fibromes pleuraux. Étude des insulin-likegrowthfactors (IGF) et considérations pathogéniques La revue de médecine interneVolume 23, numéro 5,pages 447-453 (mai 2002)

[6] H. KABIRI, L. ACHACHI, N. MAHASSINI, S. AL AZIZ, A. EL MASLOUT, F. MANSOURI, A. BENOSMAN LE MESOTHELIOME FIBREUX DE LA PLEVRE A PROPOS D'UNE OBSERVATION Médecine du Maghreb 1999 n 77

[7] TROMBATI N, BAHLAOUI A, CHERKAOUI O, BOUAYAD Z.Le mésothéliome pleural fibreux a propos d'une observation. Revue de Pneumol-clin $1994 ; 50 ; 4 ; 161-164$

[8] GUIBERT B., FILARTIGAA., DESPIERRES G., LATARJET M.Les tumeurs pleurales localisées. A propos de cinq nouveaux cas.Lyon Chir., 1978, 74, $12-16$ 
[9] ENGLAND DM., HOCHOLZER L., MC CARTHY M.J.Localised benign and malignant fibrous tumour of the pleura. A clinicopathologicalreview of 223 cases.Ain. J. Surg. Pathol., 1989, 13, 640-658

[10] SCHARIFKER D., KANEKO M.Localized fibrous mesotheliomaof pleura (submesotlielial fibroma). A clinicopathologic study of 18 cases.Cancer, 1979, 43, 627-635

[11]MORO D., BRAMBILLA E. , BRICHON PY., CLARAZ C.,COULOMB M., SARRAZIN R., PARAMELLE B., BRAMBILLA C.Les fibromespleurauxbénins. Etude anatomo-clinique de 10 cas.Rev. Mal. Respir., 1990, 7, 231-238. cases with immunohistochemicalstudy.Arch. Anat, Cytol. Math, 1993, $41, n^{\circ} 5-6,241-244$

[12] RUFFIE P.Traitement des tumeurs pleurales.Rev. Prat., 1990, 40, $1851-53$

[13] MERLIER M. LE BRIGAND H. WAPLER C.Chirurgie des tumeurs pleurales primitives.a Poumon. 1968, 24, 521-27

[14] DUBOST CL, CHARBONNIER J. Y, ASSENSP, CARIN B., JEUNEHOMME $G$, LAVERGNE A, ABELANET R, LeCHARPENTIER Y.Fibromes géants de la plèvre. A propos de deux observations.J. Chir.(Paris), 1984, 121, 175-81 